\title{
Glomerular Podocytes Express Type 1 Adenylate Cyclase: Inactivation Results in Susceptibility to Proteinuria
}

\author{
Zhijie Xiao $^{a}$ Liqun He $^{a}$ Minoru Takemoto ${ }^{a, d}$ Hannu Jalanko ${ }^{e}$ Guy C. Chan ${ }^{f}$ \\ Daniel R. Storm ${ }^{f}$ Christer Betsholtz ${ }^{a, b} \quad$ Karl Tryggvason ${ }^{a} \quad$ Jaakko Patrakkaa, c \\ ${ }^{a}$ Division of Matrix Biology, Department of Medical Biochemistry and Biophysics, Karolinska Institute, \\ and Departments of ${ }^{\mathrm{b}}$ Medicine and ${ }^{\mathrm{c}}$ Renal Medicine, Karolinska University Hospital, Stockholm, Sweden;

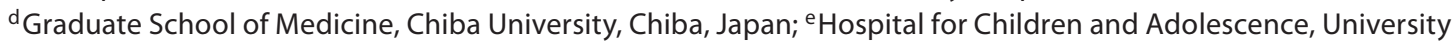 \\ of Helsinki, Helsinki, Finland; ' Department of Pharmacology, University of Washington, Seattle, Wash., USA
}

\section{Key Words}

Adc1 · Adenylate cyclase • Albuminuria • Glomerular

podocytes $\cdot$ Proteinuria

\begin{abstract}
Background/Aims: The organization of actin cytoskeleton in podocyte foot processes plays a critical role in the maintenance of the glomerular filtration barrier. The CAMP pathway is an important regulator of the actin network assembly in cells. However, the role of the CAMP pathway in podocytes is not well understood. Type 1 adenylate cyclase (Adcy1), previously thought to be specific for neuronal tissue, is a member of the family of enzymes that catalyses the formation of CAMP. In this study, we characterized the expression and role of Adcy1 in the kidney. Methods: Expression of Adcy1 was studied by RT-PCR, Northern blotting and in situ hybridization. The role of Adcy1 in podocytes was investigated by analyzing Adcy1 knockout mice (Adcy1-/-). Results and Conclusion: Adcy1 is expressed in the kidney specifically by podocytes. In the kidney, Adcy1 does not have a critical role in normal physiological functioning as kidney histology and function are normal in Adcy1-/- mice. However, albumin overload resulted in severe albuminuria in Adcy1-/- mice,
\end{abstract}

whereas wild-type control mice showed only mild albumin leakage to urine. In conclusion, we have identified Adcy1 as a novel podocyte signaling protein that seems to have a role in compensatory physiological processes in the glomerulus.

Copyright $\odot 2010$ S. Karger AG, Basel

\section{Introduction}

Podocytes play a critical role in the maintenance of the normal glomerular filtration barrier [1]. This is highlighted by the fact that mutations in podocyte proteins result in hereditary proteinuria syndromes [2-8]. Foot processes of podocytes envelope glomerular capillary loops and form the final barrier for filtration [9]. Basally, foot processes are embedded in the glomerular basement membrane (GBM), and laterally adjacent foot processes are connected by a specialized podocyte-podocyte cell junction, the slit diaphragm. The cytoskeleton of foot processes is characterized by the presence of highly ordered parallel contractile actin filament bundles. This actin-based cytoskeleton connects three membrane domains of podocytes, namely the apical membrane, the slit diaphragm and the basal membrane domain. The

\section{KARGER}

(C) 2010 S. Karger AG, Basel

Fax +4161306 1234 E-Mail karger@karger.ch www.karger.com www.karger.com/nee
Jaakko Patrakka, MD, $\mathrm{PhD}$

Division of Matrix Biology

Department of Medical Biochemistry and Biophysics

Karolinska Institute, SE-171 77 Stockholm (Sweden)

Tel. +46 831 3445, Fax +46 76205 1933, E-Mail jaakko.patrakka@ki.se 
central role of the actin cytoskeleton is highlighted by the fact that defects in any of these membrane domains lead to dramatic changes in the actin cytoskeleton organization [10]. Instead of the normal parallel contractile bundles, these changes in cytoskeletal organization lead to the formation of a dense network that is observed as foot process effacement. Foot process effacement is, on the other hand, usually associated with proteinuria, and is basically observed in most human proteinuric diseases. Therefore, knowledge on the molecular machinery regulating the assembly of actin cytoskeleton in foot processes is one of the central issues in glomerular biology.

A major player in the regulation of actin assembly in cells is the cyclic AMP (cAMP) pathway, which conducts signals from the plasma membrane-bound G-protein-coupled receptors (GPCR) to the actin cytoskeleton [11]. Ligands binding to GPCRs activate adenylate cyclases (ACs), which catalyze the formation of CAMP from ATP. The role of the cAMP pathway in podocytes is not well understood [12]. Several receptors whose stimulation in podocytes results in cAMP pathway activation, such as prostaglandin receptors IP and EP4, have been identified [12-15]. The downstream effects of cAMP in podocytes have been suggested to include regulation of cell morphology and actin assembly $[12,16]$. However, direct evidence of the importance of the CAMP pathway in podocyte biology is lacking.

In this study, we sought to identify components of the cAMP pathway important for podocyte biology and the filtration barrier. We show that type 1 AC (Adcyl), a unique $\mathrm{AC}$ isoform previously thought to be specific for neuronal tissue, is also expressed in glomerular podocytes. Furthermore, we show that Adcyl has an important role in regulating the glomerular filtration barrier function as Adcyl-deficient mice are susceptible to develop proteinuria under pathological stimulus.

\section{Methods}

Analysis of AC Isoform Expression Profiles in the Glomerulus

Glomerular expression profiling using the glomerular transcript database (GLOMBASE) and Affymetrix chips has been described previously [17]. Briefly, we generated GLOMBASE by sequencing approximately 15,000 expressed sequence tags from mouse glomerular cDNA libraries. These clones were amplified and anchored to glass slides and used for a series of hybridization experiments. The expression profiles of GLOMBASE clones were analyzed by comparing transcriptomes of the glomerular fraction composed of podocytes to the non-podocyte portion of the glomerulus. In addition, Affymetrix analyses were performed to compare the expression levels between the glomerulus fraction and the kidney fraction devoid of glomeruli.
Expression of Adcy1 Transcript in Various Mouse Organs

The expression of Adcyl mRNA in various mouse organs was studied using RT-PCR and Northern blotting. Adcyl-specific oligonucleotides (L-CAGCACTTCCTCATGTCCAA, R-CCAGTGCTATCCATCCGACT) for PCR analysis were designed to amplify a 500-bp product. As a template, we used cDNA generated from different adult mouse tissues (Mouse Multiple Tissue cDNA Panel I, Clontech Laboratories, Palo Alto, Calif., USA). For Northern blotting, the 500-bp PCR product obtained from amplification of a brain cDNA library was used as the cDNA probe. The probe was ${ }^{32} \mathrm{P}$ labeled and hybridized to the Multiple Tissue Northern (MTN) blot containing RNAs isolated from various mouse organs according to manufacturer's instructions (Clontech Laboratories).

\section{In situ Hybridization}

In situ hybridizations were performed on paraffin-embedded tissue sections $(10 \mu \mathrm{m})$ collected from 4 -week-old and newborn mouse kidneys as described previously [18]. The probes for in situ hybridization were synthesized by amplifying a 500-bp cDNA fragment corresponding to the 3'UTR end of the mouse Adcy1 gene from a brain cDNA library (see above) and by subcloning this product into the pCR II-TOPO Dual Promoter Vector (Invitrogen, Carlsbad, Calif., USA). Antisense and sense probes were prepared using T7 and SP6 polymerases.

\section{Analysis of Adcy1 Knockout Mice}

To study the role of Adcyl in vivo, we analyzed Adcyl knockout (Adcyl-/-) mice generated previously by $\mathrm{Wu}$ et al. [19]. The Adcyl-/- mice used in our study were backcrossed to C57/Bl6 background $>10$ generations. All animal experiments performed were approved by the local ethics committee.

For morphological analysis, the samples were prepared according to standard procedures. Light microscopy was performed on newborn, 10-week-old and 1-year-old Adcyl-/- kidneys ( $\mathrm{n}=$ 5/age group). For light microscopy, we prepared kidneys from 3 littermate control mice as a control. For electron microscopy, samples from 10-week-old and 1-year-old Adcy1-/- kidneys ( $\mathrm{n}=$ 3 , respectively) were prepared. For electron microscopy, kidney samples from 3 littermate control mice were prepared as controls.

To study the expression of podocyte proteins in the Adcyl knockout mice, we immunostained the knockout and littermate control kidneys with anti-synaptopodin (Progen, Heidelberg, Germany), anti-nephrin [20], anti-podocin [21], anti-wt1 (Santa Cruz Bitotechnology, Santa Cruz, Calif., USA) and anti-dendrin [22] antibodies. The stainings were performed on frozen kidney sections collected from 8-week- and 1-year-old mice as described previously [22].

Beside immunostaining, we performed RT-PCR experiments to analyze the expression of different AC isoforms in wild-type and knockout glomeruli. Oligonucleotides used for the amplification and the sizes of expected PCR products were as follows: Adcy2 L-ATCTCCTGCTCCGTTTTCCT, R-ATGTATTGACGCTCGGGTTC, 659 bp; Adcy3 L-CATCGAGTGTCTACGCTTC, R-GGATGACCTGTGTCTCTTCT, 443 bp; Adcy4 L-GGAGCGAGAGGAGACTGAGA, R-GGCGGAAGTTATTGAACGAG, 470 bp; Adcy5 L-TGGAACTCAAGGGTCTGCTT, R-TGGGATGTGTGTAAGGTGGA, 606 bp; Adcy6 L-CTGCTTGTGTTCATCTCTCTG, R-GACGCTAAGCAGTAGATCA, 365 bp; Adcy7 LCCAGTTATTTAGAGAGAGACCTG, R-CTTGCTCATCAGG-
Xiao/He/Takemoto/Jalanko/Chan/ Storm/Betsholtz/Tryggvason/Patrakka 
Table 1. Expression of AC isoforms in the kidney by microarray analysis

\begin{tabular}{llll}
\hline AC isoform & \multicolumn{2}{l}{ Glomerulus/rest of the kidney } & \multirow{2}{*}{$\begin{array}{l}\text { Podocyte/ } \\
\text { non-podocyte }\end{array}$} \\
\cline { 2 - 3 } & 4 months & 5 days & \\
\hline Adcy1 & 7.98 & 5.64 & 5.2 \\
Adcy2 & NS & NS & \\
Adcy3 & 4.56 & NS & NS \\
Adcy4 & 35.93 & 6.65 & NS \\
Adcy5 & 5.07 & NS & \\
Adcy6 & NS & NS & \\
Adcy7 & 2.97 & NS & NS \\
Adcy8 & NS & NS & \\
Adcy9 & NS & NS & \\
\hline
\end{tabular}

The expression levels in the glomerulus were compared to that of the rest of the kidney, and the glomerulus fraction was further subdivided to podocyte and non-podocyte parts. The experiments were performed in 4-month-old and 5-day-old (developing) kidneys. The levels are expressed as ratios of glomerulus/rest of the kidney and podocyte/non-podocyte fraction. NS = Nonsignificant.

GCCATGCTAA, 560 bp; Adcy8 L-CGATGCTGTTGGAGTGATGT, R-AAGATGAATGGGTTGGGTTG, 609 bp, and Adcy9 L-AGCTTATCCTCACCTTCTTCTTCCTC, R-GGCAAGGAGTGCTACCGTGTCCT, 314 bp. As a template, we used cDNAs generated from glomerular mRNAs isolated from Adcyl-/- and wild-type mice. The glomerular portions were isolated as described previously [17]. As positive control templates, we used cDNA libraries generated from mRNAs isolated from different adult mouse tissues (Mouse Multiple Tissue cDNA Panel I; Clontech Laboratories). For each AC isoform, we used a tissue that had previously been shown to express the corresponding isoform (Adcy2-lung, Adcy3-brain, Adcy4-brain, Adcy5-heart, Adcy6lung, Adcy7-brain, Adcy8-lung and Adcy9-muscle) [22].

We also performed quantitative real-time PCR for different $\mathrm{AC}$ isoforms to evaluate expressional changes in Adcyl-deficient glomeruli more precisely. Reverse transcription was performed using the Superscript III first-strand synthesis system for RT-PCR (Invitrogen). TaqMan gene expression assays from Applied Biosystems (Foster City, Calif., USA) were performed using standard methodology, which included the use of murine glyceraldehyde3-phosphate dehydrogenase as a control.

\section{Induction of Proteinuria in Adcyl-Deficient Mice}

To study how Adcyl-deficient mice respond to pathological stimuli, we induced proteinuria using bovine serum albumin (BSA) overload and lipopolysaccharide (LPS) proteinuria models. In these experiments, we used female mice. BSA overload was generated by daily injection of BSA $(400 \mu l, 0.5 \mathrm{~g} / \mathrm{ml}$ i.p.; Merck, Darmstadt, Germany) on 4 consecutive days. Altogether, 10 Adcyl-/- and 10 littermate 10-week-old mice were included in the protein overload experiment. To follow the outcome of the protein overload experiment, urine samples were collected before each intraperitoneal injection and every $24 \mathrm{~h}$ after the last injection.
Histological analysis of mice in the protein overload experiment was performed 3 and 6 days after the first injection for 3 Adcyl-/mice and 3 control animals at each time point. Samples for histological analysis were prepared as described above. The degree of foot process effacement was analyzed by counting the number of filtration slits/GBM length $(100 \mu \mathrm{m})$. For the LPS experiment, we gave a single intraperitoneal injection of LPS $(200 \mu \mathrm{g}$ in a total volume of $200 \mu \mathrm{l}$, Sigma-Aldrich). Urine was collected at the time of LPS injection, and 12, 24, 36, 48, 60 and $72 \mathrm{~h}$ after the injection. Also, the expression of podocyte proteins synaptopodin, podocin, wt 1 , nephrin and dendrin was studied on days 3 and 7 using immunofluorescence staining as described above.

Urine Analysis

Urine samples collected from Adcyl-/- and control mice were analyzed in two different ways. The amount of albuminuria was measured with ELISA for albumin according to the manufacturer's instructions (Albuwell M; Exocell, Philadelphia, Pa., USA). The quality of proteins in the urine was analyzed by loading $1 \mu \mathrm{l}$ of urine sample on 4-20\% polyacrylamide gel (Ready Gels, BioRad Laboratories, Hercules, Calif., USA) and by staining the gels with Coomassie brilliant blue R-250 (Bio-Rad Laboratories, Hercules, Calif., USA) using standard methodology.

\section{Results}

\section{Expression of AC Isoforms in the Kidney Using Microarray Analysis}

In microarray experiments using Affymetrix chips, we observed a significant enrichment ( $>2$-fold) of Adcyl, Adcy3, Adcy4, Adcy5 and Adcy7 in the glomerular fraction compared to the rest of the kidney (table 1). In the glomerular expression library (GLOMBASE), only Adcyl, Adcy 4 and Adcy 6 were present. Of these, Adcyl had eight copies of expressed sequence tags, whereas Adcy4 and Adcy6 had no more than one and two copies, respectively. These GLOMBASE clones were analyzed further by microarray in which the expression level of podocytes was compared to the non-podocyte fraction of the glomeruli. Of these three AC isoforms, only Adcyl was significantly enriched in podocytes (table 1).

\section{Expression of Adcyl in Various Mouse Organs}

As our microarray data suggested that Adcyl was present in the podocyte transcriptome, we decided to investigate the expression profile of the $A d c y 1$ gene in more detail. First, expression of the Adcyl gene was evaluated in various mouse organs by RT-PCR and Northern blotting. RT-PCR experiments amplified an expected 500-bp fragment in RNA isolated from brain and kidney tissues, whereas other tissues gave no signal (fig. 1a). In Northern blotting, the probe for Adcyl hybridized to an mRNA sized $\sim 11.5 \mathrm{~kb}$ in mRNAs isolated 


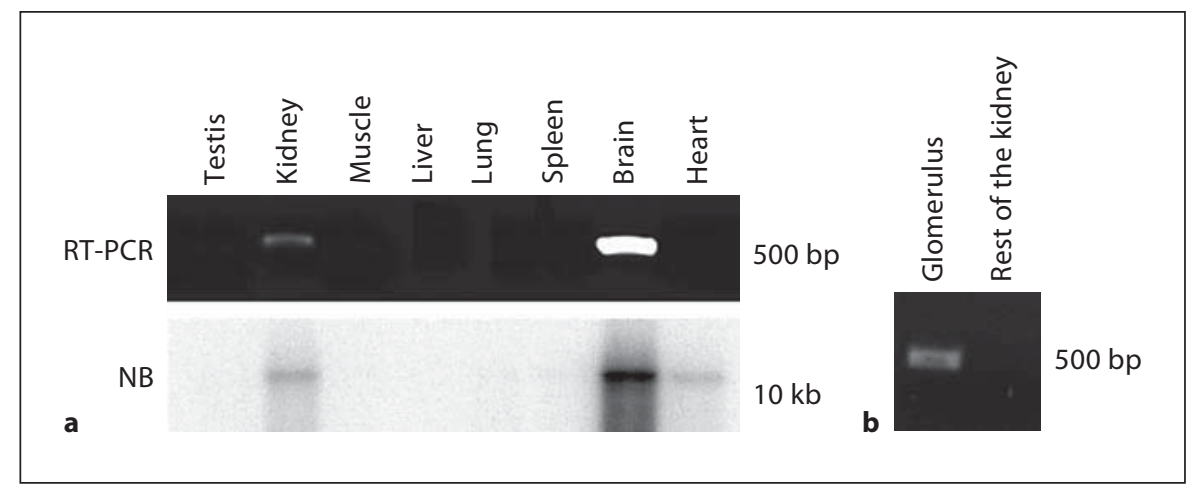

Fig. 1. Expression of Adcyl transcript in various mouse organs detected by RT-PCR and Northern blotting (NB). a RT-PCR analysis of RNA isolated from different mouse organs shows that Adcyl transcript can be found only in the brain and kidney as an expected 500-bp product is amplified from these tissues. In
Northern blotting, the Adcyl probe hybridizes to an mRNA around $11.5 \mathrm{~kb}$ in RNA extracted from brain, kidney and heart tissues. The signal for Adcyl mRNA is strongest in the brain. No signal is detected in other tissues. b In the kidney, Adcyl transcript is detected solely in the glomerulus fraction by RT-PCR. from brain, heart and kidney tissues, while no signal was detected in other tissues (fig. 1a). In order to further define which portion of the kidney expressed the Adcyl transcript, we performed RT-PCR experiments on kidney fractions containing either glomerular fraction or the kidney portion lacking glomeruli. Adcyl transcript was detected exclusively in the glomerular fraction of the kidney by RT-PCR (fig. 1b). Thus, the presence of Adcyl transcript was confirmed in kidney tissue using both RT-PCR and Northern blotting.

\section{Expression of Adcyl Transcript in the Kidney by in situ Hybridization}

As RT-PCR and Northern blotting experiments confirmed the presence of Adcyl transcript in the kidney, we performed in situ hybridization for Adcyl on kidney sections collected from 4-week-old and newborn mice. In the adult kidney, the antisense probe for $A d c y 1$ mRNA gave signal in glomerular capillary tufts, and outside glomeruli only background signal was detected (fig. 2a, b). The signal concentrated to the periphery of glomerular capillary loops suggesting localization to podocytes. Next, we analyzed the expression of Adcyl transcript in glomerulogenesis during which glomerular cells are more distinctively recognizable. At the capillary stage of the developing glomerulus, pre-podocytes are organized as a line in the periphery of maturing capillary loops. During this stage, the signal for Adcyl mRNA was clearly localized to pre-podocytes along the periphery of the developing glomerulus (fig. 2c). Experiments with Adcyl sense probe showed only background signal (fig. $2 \mathrm{~d}$ ). No
Adcyl expression was detected in vesicle and S-shaped stage glomeruli (data not shown).

\section{Kidney Phenotype of Adcy1 Knockout Mice}

To investigate the role of Adcyl in podocytes in vivo, we analyzed the kidney phenotype of Adcyl knockout mice. Kidney histology was evaluated in newborn, 8-week-old and 1-year-old Adcy1-/- mice. Light-microscopic evaluation showed normal kidney histology and glomerular morphology in all age groups (fig. 3a). In electron microscopy, podocytes showed normal morphology with regularly interdigitating foot processes, which were connected by intact slit diaphragms (fig. 3b, c). The expression of the podocyte proteins nephrin, podocin, synaptopodin, podocalyxin, wt 1 and dendrin was unchanged in Adcyl-/- mice, as detected by immunofluorescence staining (data not shown). To evaluate the kidney filter function, we analyzed albumin levels in urine from 10 week- and 1-year-old Adcy1-/- mice. Urine analysis showed no significant proteinuria in Adcyl knockout mice using albumin-ELISA or SDS-PAGE analysis (data not shown).

As no obvious renal phenotype in Adcyl-deficient mice was observed, possible compensatory changes were investigated in the expression levels of other AC isoforms in glomeruli of Adcyl knockout animals. For this, we performed RT-PCR experiments from isolated glomerular tufts. Different AC isoforms seemed to be expressed at approximately same levels in Adcyl-deficient and control animals as detected by RT-PCR analysis (fig. 3d). Control PCR for G3PDH gave similar signal in both wild- 

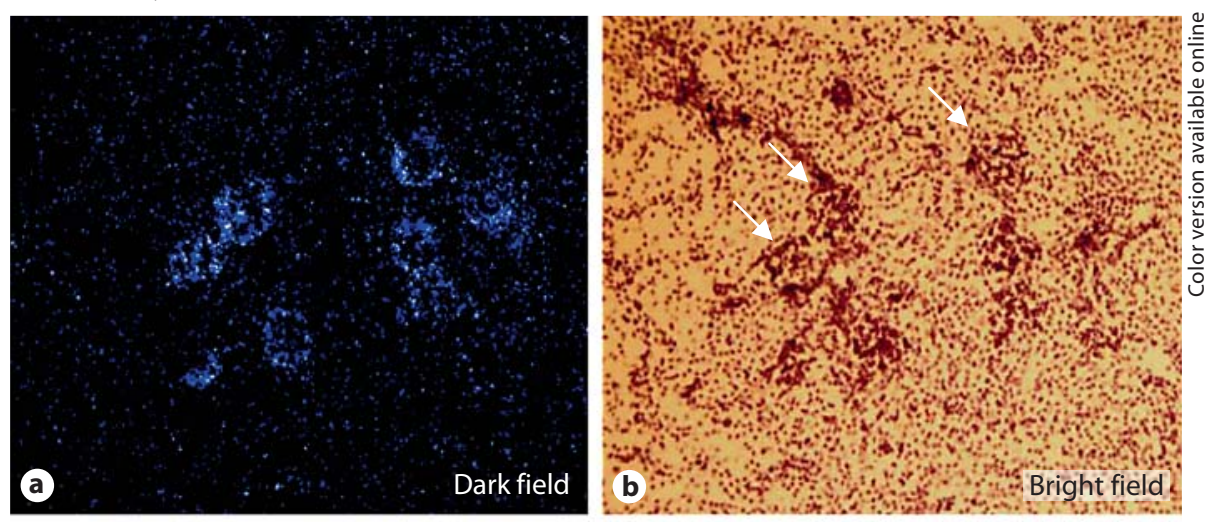

Fig. 2. Expression of Adcyl transcript in adult and developing mouse kidney detected by in situ hybridization. a, b In adult kidney, in situ hybridization with Adcyl antisense probe reveals signal for Adcyl mRNA in glomeruli (corresponding bright and dark field images). Only background signal is detected outside glomeruli in kidney tissue. Arrows indicate glomeruli in the kidney cortex. c At the capillary stage of the glomerulus, the signal for Adcyl transcript is concentrated to the periphery of developing capillary loops where prepodocytes are localized. $\mathbf{d}$ The sense probe gives only background signal. Dashed lines surround the urinary spaces of developing glomeruli. $\times 80(\mathbf{a}, \mathbf{b}) . \times 300(\mathbf{c}, \mathbf{d})$.
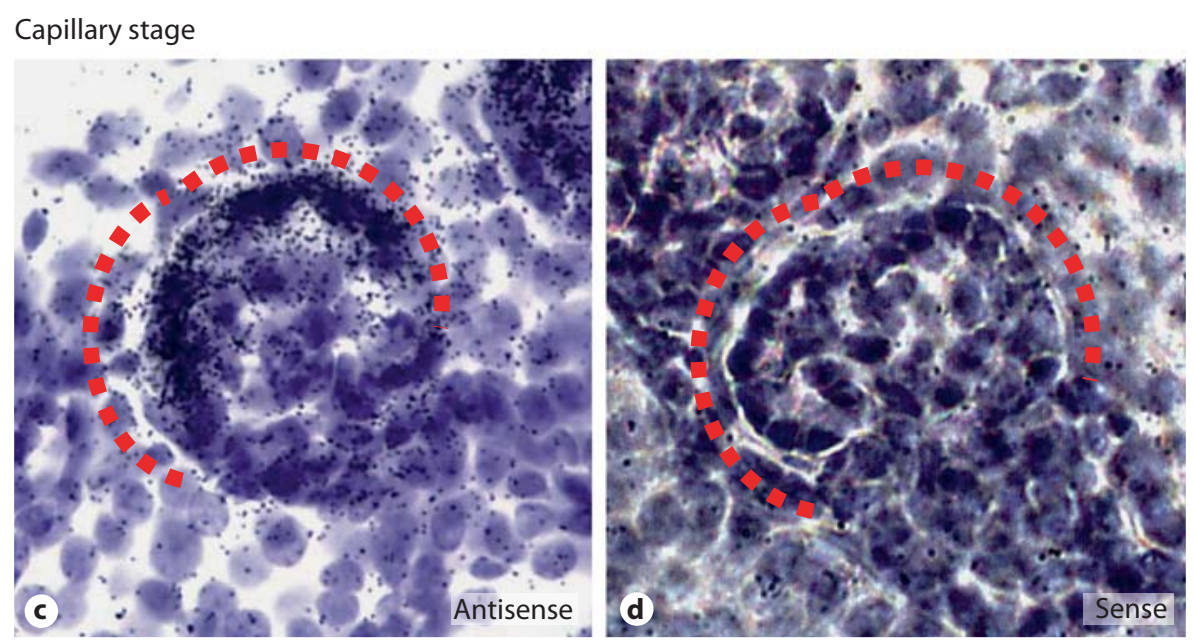

type and knockout glomeruli. As positive controls, we performed RT-PCR experiments on mRNAs isolated from control tissues known to express different $\mathrm{AC}$ isoforms (fig. $3 \mathrm{~d}$ ). To gain more quantitative data, we performed real-time PCR experiments for AC isoforms. The expression of other AC isoforms was not significantly changed between wild-type and knockout glomeruli as detected by this quantitative assay (Mann-Whitney $\mathrm{U}$ test, data not shown). Thus, the lack of Adcyl in knockout animals does not seem to be compensated by increased expression of other $\mathrm{AC}$ isoforms in the glomerulus.

\section{Response to Pathological Stimuli in Adcyl Knockout \\ Mice}

Due to the apparent absence of glomerular defects in Adcyl-/- mice, we induced a pathological condition by protein overload and LPS injection. For the overload experiment, intraperitoneal injections of BSA were administered on 4 consecutive days. The dose was titrated in initial experiments so that the albuminuria induced in wild-type animals was low. Based on this, 10-week-old mice were injected with $2 \mathrm{mg}$ of BSA on 4 consecutive days.

The level of albuminuria in wild-type littermate control mice slightly increased during the first day of induction of protein overload $(0.12 \pm 0.25 \mathrm{~g} / \mathrm{ml})$ but returned to baseline after that (fig. $4 \mathrm{a})$. In electron-microscopic examination, podocytes in control mice showed normal morphology under the protein overload as regularly interdigitating foot processes were surrounding the GBM (fig. 4c). In contrast to this, Adcyl-/- mice injected with BSA developed massive albuminuria $24 \mathrm{~h}$ after the first injection (day 2: $0.94 \pm 0.57 \mathrm{~g} / \mathrm{ml}$ ), and the level of albuminuria remained high for 4 days (day 3: $0.96 \pm 0.41 \mathrm{~g} / \mathrm{ml}$; day 4: $0.68 \pm 0.47$ $\mathrm{g} / \mathrm{ml}$, and day 5: $0.60 \pm 0.42 \mathrm{~g} / \mathrm{ml}$; fig. 4a). Within 2 days after the last BSA injection, albuminuria in Adcyl-/- returned to the normal baseline. Urine analysis on SDSPAGE gel revealed the presence of high amounts of albumin in the Adcyl-/- mouse urine, whereas only very low amounts of albumin were detected in littermate controls 
Fig. 3. Phenotypical analysis of Adcyl-/kidneys. a HE-stained kidneys collected from 10-week-old Adcyl-/- mice show normal kidney histology and glomerular morphology $(\times 150, \times 400)$. b, c In electron microscopy, the podocyte morphology is normal with fine interdigitating foot processes, which are connected by intact slit diaphragms (arrowheads, scale bars: 200 and $800 \mathrm{~nm}$ ). d RT-PCR analysis of glomerular mRNAs isolated from Adcyl-/and wild-type mice. Expression of Adcy3, Adcy4, Adcy5, Adcy6 and Adcy9 is detected in glomeruli isolated from knockout mice. In comparison to glomeruli collected from wild-type mice, no clear difference is observed in expression levels of these AC isoforms by RT-PCR. G3PDH, used as a loading control, shows similar expression in both glomerular samples. Control RT-PCR experiments performed on mRNAs isolated from tissues known to express different AC isoforms give expected PCR fragments (Adcy2-lung, 659 bp; Adcy3-brain, 443 bp; Adcy4-brain, 470 bp; Adcy5-heart, 606 bp; Adcy6-lung, 365 bp; Adcy7-brain, 560 bp; Adcy8-lung, 609 bp, and Adcy9-muscle, 314 bp).

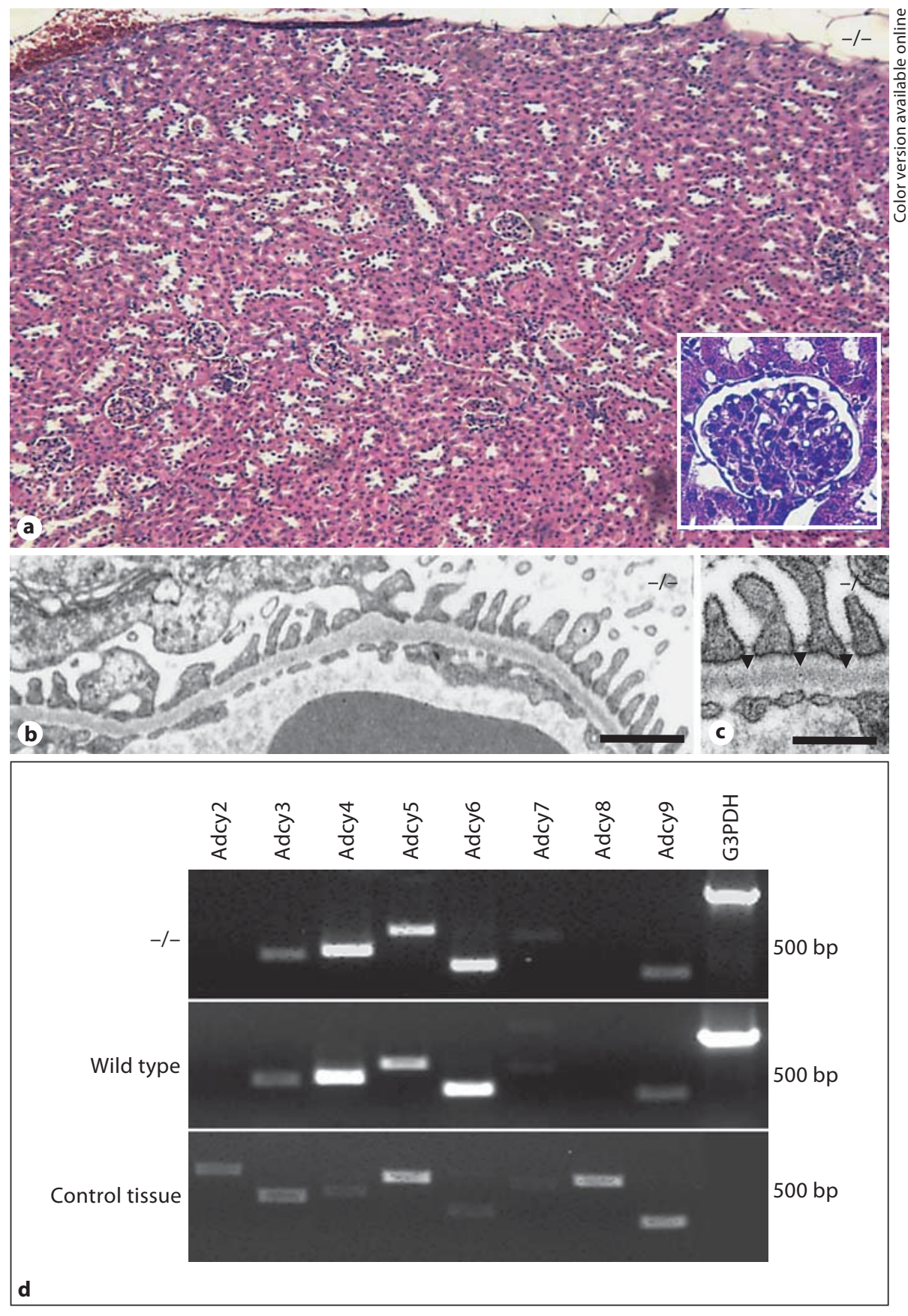

(fig. 4b). Of note, the albumin overload resulted in nonselective proteinuria as proteins larger than albumin were also observed on SDS-PAGE analysis. Electron-microscopic analysis in Adcy1-/- mice with albumin overload showed abnormalities in podocyte structure. Most areas of the GBM were surrounded by regular-looking thin foot processes in the knockout mice but rather often areas of local foot process effacement were observed (fig. 4c). The degree of foot process effacement was significant $(\mathrm{p}<0.05$, Mann-Whitney test) in Adcyl-/- mice as the number of slits/GBM length was decreased from 303/100 $\mu \mathrm{m}$ (controls) to $187 / 100 \mu \mathrm{m}$ (Adcyl-/-). Occasionally, vacuoles were also observed in podocytes of Adcyl-/- mice during the protein overload experiment (data not shown). Expressional changes of the podocyte proteins nephrin, podocin, dendrin, synaptopodin and wt1 were investigated on days
Xiao/He/Takemoto/Jalanko/Chan/ Storm/Betsholtz/Tryggvason/Patrakka 
Fig. 4. Urine and morphological analysis of the protein overload experiment. Five Adcyl-/- and 5 control mice were injected with BSA on 4 consecutive days (days 1-4), and urine was collected at the time of each injection and every $24 \mathrm{~h}$ after the last injection. Black bars indicate wild-type samples, white bars Adcyl-/- samples. a No significant albuminuria was observed before the first BSA injection (day 1). In wildtype mice, moderate levels of albumin $(0.12 \pm 0.25 \mathrm{~g} / \mathrm{ml})$ were detected in urine samples collected $24 \mathrm{~h}$ after the first injection. No albuminuria was detected in wild-type mice after day 2 . In contrast, Adcyl-/- showed massive albuminuria as early as $24 \mathrm{~h}$ after the first injection (day 2: $0.94 \pm 0.57 \mathrm{~g} / \mathrm{ml}$ ), and albumin leakage remained massive for the 4 days: day 3 : $0.96 \pm 0.41 \mathrm{~g} / \mathrm{ml}$, day $4: 0.68 \pm 0.47 \mathrm{~g} / \mathrm{ml}$, day 5: $0.60 \pm 0.42 \mathrm{~g} / \mathrm{ml}\left({ }^{*} \mathrm{p}<0.05\right.$, t test). b Urine analysis on SDS-PAGE gel. One microliter of urine (collected on day 3 ) was run on gel. A strong band around $65 \mathrm{kDa}$ is present in the Adcyl samples indicating massive albuminuria. Larger proteins are also detected in the urine of Adcyl-/mice. In contrast, littermate controls show only minimal albuminuria in the gel analysis. Data from individual mice are shown. c During the protein overload, podocyte foot processes are occasionally effaced in glomeruli of Adcyl-/- mice, whereas the wild-type mice show normal podocyte morphology under this pathological stimulus (scale bars: $600 \mathrm{~nm}$ ). wt = Wild type.
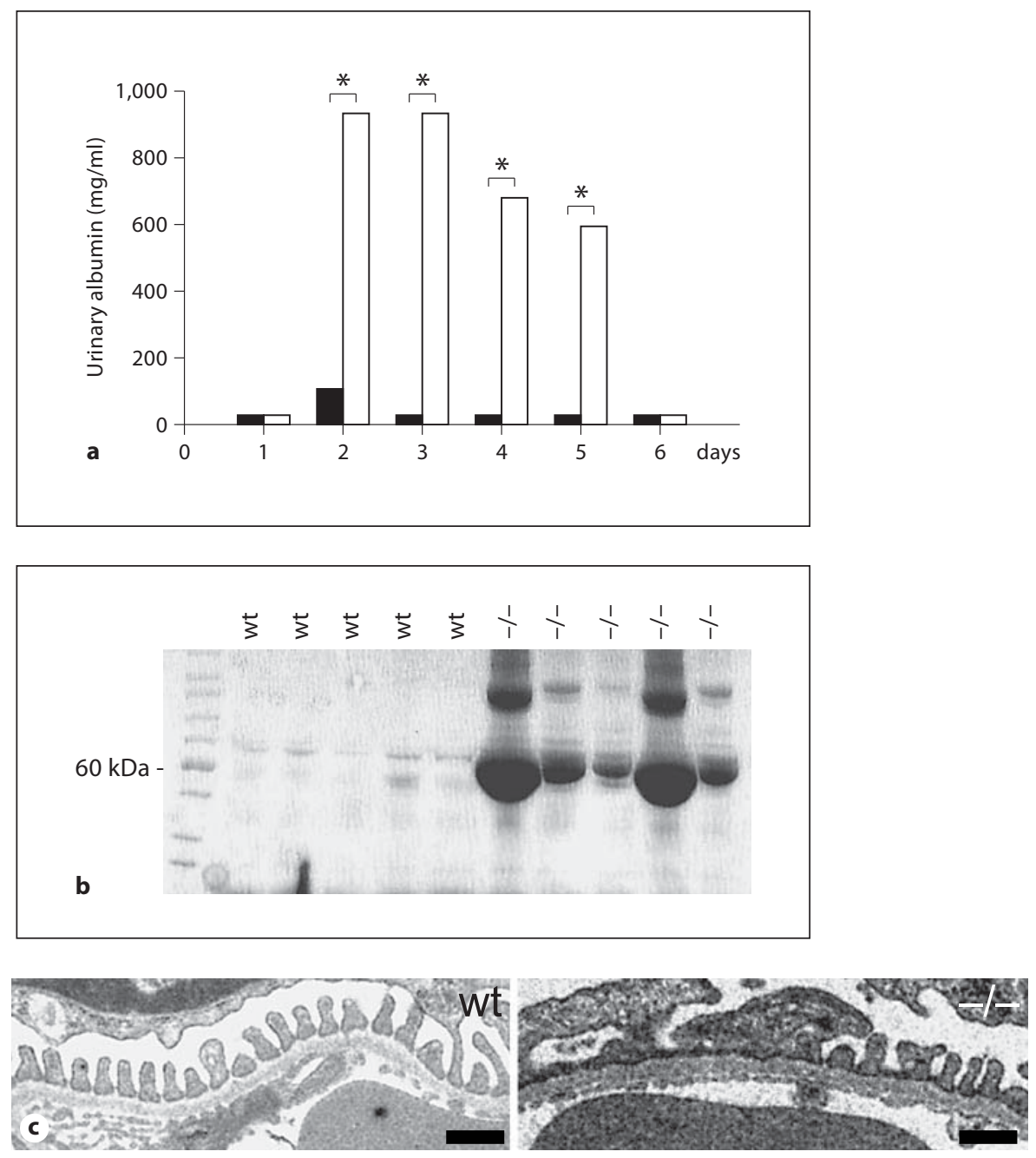

3 and 7 using immunostaining. We did not observe any obvious changes in the staining intensity for these proteins in wt and Adcyl-/- mice injected with BSA (fig. 5, data not shown). However, the staining pattern for the foot process proteins nephrin, podocin, dendrin and synaptopodin was altered from a thin sharp line of reactivity to a slightly more thicker (more diffuse) staining in Adcyl-/- mice injected with BSA (fig. 5, data not shown). This change was probably secondary to the foot process effacement observed in these mice.

A single intraperitoneal injection of LPS resulted in transient proteinuria, as described previously [23]. However, in contrast to the protein overload experiment, we did not observe any significant differences in the amount of albuminuria between Adcyl knockout mice and control animals in the LPS-induced proteinuria model (data not shown).

Type 1 Adenylate Cyclase and Proteinuria

\section{Discussion}

The cAMP pathway is activated by extracellular ligands that bind to GPCRs, which turn on ACs to catalyze the formation of cAMP. In mammals, there are nine isoforms of ACs (types 1-9). Some of these are widely expressed, whereas others (such as Adcyl) are more tissue specific. Individual AC isoforms have distinct properties which together with the differential expression allow them to interpret and integrate signals in signal transduction. The importance of different $\mathrm{AC}$ isoforms is highlighted by the fact that the inactivation of individual isoforms in the mouse results in various phenotypes [24]. In the kidney, Bek et al. [25] have previously analyzed the expression of different AC isoforms. They detected the presence of seven $\mathrm{AC}$ isoforms in the kidney glomerulus by RT-PCR. They used immunohistochemistry to local- 
Fig. 5. Expression of the podocyte foot process proteins podocin and dendrin in BSA overload as detected by immunofluorescence staining. In wild-type (wt) mice injected with BSA (day 3), podocin and dendrin are detected as a sharp thin linear line surrounding glomerular capillary loops. In Adcyl-/- mice, the staining intensity for podocin and dendrin is not clearly altered after the induction of BSA overload (days 3 and 7). However, the staining pattern is altered to a slightly thicker line of reactivity in Adcyl-/- mice. $\times 300$.
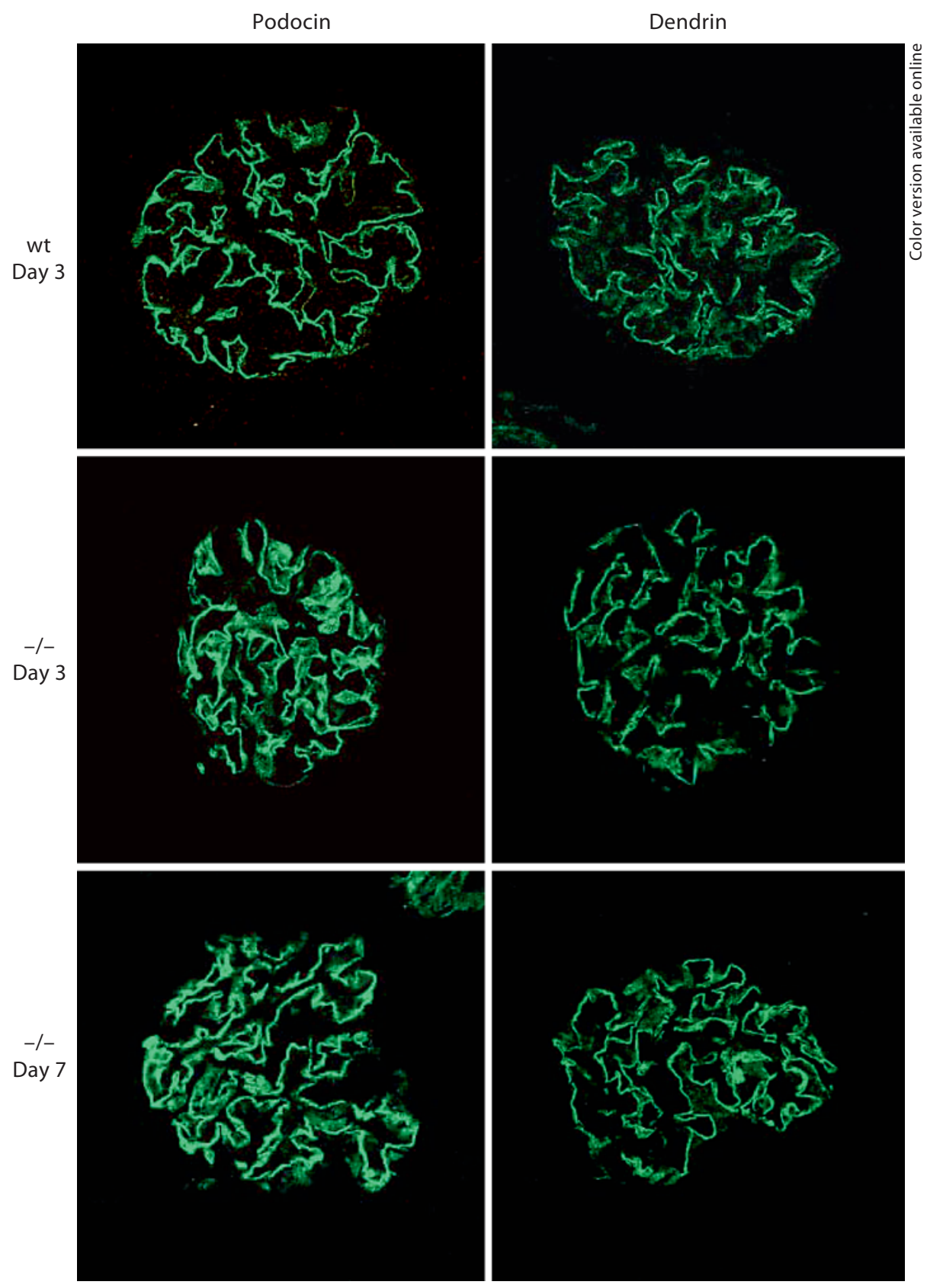

ize $\mathrm{AC}$ isoforms in the kidney in more detail. However, they did not analyze the expression of Adcyl in the kidney. The anti-AC antibodies used in that study might show cross-reactivity with different $\mathrm{AC}$ isoforms, and therefore those results should be evaluated carefully. In our hands, several commercial antibodies directed against specific AC isoforms have shown cross-reactivity with other isoforms when used in knockout tissue (data not shown). In the present approach, we analyzed our previous microarray data [17] and found that the AC isoform predominating in the podocyte transcriptome was Adcyl. We detected this podocyte expression also by in situ hybridization, and found that Adcyl is in the kidney expressed exclusively in podocytes. Outside the kidney, Adcyl expression was detected in the brain and the heart. In the brain, Adcyl has been found in certain neuronal cells [11], and Adcyl can be therefore added to a group of proteins that are shared by neuronal and podocyte cells, 
such as synaptopodin, dendrin and components of synaptic vesicles [22, 26, 27]. It is interesting to reflect on the similarity of these two cell types as both have long slender cellular processes that are capable of structural reorganization reasonably quickly (plasticity).

The role of the cAMP pathway in podocyte biology is not well understood. Several receptors whose stimulation result in increased cAMP levels in podocytes have been identified [12-15]. Downstream effects of cAMP in podocytes have been shown to include activation of protein kinase A (PKA) and depolarization via chloride channels [13]. Also, the cAMP pathway has been linked to the cellular redox state in podocytes as Stra13, a prostaglandin $\mathrm{E}_{2}$-induced gene, seems to protect podocytes from oxidative stress [28]. In addition, the cAMP pathway has been linked to the regulation of cell morphology, actin assembly and matrix production in podocytes [13]. In this study, Adcyl-deficient mice showed no abnormalities in kidney morphology or filtration function. However, in response to protein overload, we observed a significant difference between knockout and control animals. While BSA injection on consecutive days resulted only in minimal proteinuria in control mice, Adcyl knockout mice developed massive proteinuria $24 \mathrm{~h}$ after the first injection. The reason for this pathological response was not analyzed further in this study. However, based on the present data, it is reasonable to speculate that the absence of Adcyl is likely to result in defective cAMP signaling in podocytes, and therefore cause an impaired activation of PKA. PKA regulates the assembly of actin cytoskeleton via actin phosphorylation. As albumin overload in Adcyl-deficient mice results in reorganization of the foot process actin cytoskeleton (effacement), we hypothesize that the abnormal podocyte response observed in Adcyldeficient mice may be the cause of impaired actin cytoskeleton assembly though defective PKA signaling. So far, we lack direct evidence to support this theory, and more studies are clearly needed to analyze whether our hypothesis is true.

The albumin overload model has been classically used for studying tubular function in handling excess filtered albumin. However, this model has been previously shown to be a valid model in analyzing susceptibility for proteinuria in mice lacking perlecan sulfate side chains, which are considered having a primary defect in the GBM [29]. As Adcyl seems to be expressed only by podocyte cells and not by the tubular epithelium, our study supports the idea that BSA overload is a useful model for challenging the glomerular filtration barrier. However, it is important to notify that the genetic background of the mice seems to modify the response to BSA overload [30]. In our study, we used mice that had been backcrossed to B57/Bl6 background. Also, we used littermate controls to eliminate the effects of genetic background. Of note, the fact that BSA- but not LPS-induced proteinuria results in impaired response in Adcyl-/- mice suggests that these two proteinuria models differ mechanistically.

To conclude, the present report is - to our knowledge - the first study that directly points to the critical role of the cAMP pathway in the glomerular filtration function. The finding that defective cAMP signaling results in susceptibility to proteinuria is very interesting, as this pathway can serve as a target for pharmacological treatment. In fact, Tam et al. [31] have previously shown that treatment with the phosphodiesterase inhibitor rolipram (which promotes cAMP activity by inhibiting breakdown of cAMP) effectively reduces proteinuria and the progression of glomerular disease in rats with crescentic glomerulonephritis. Obviously, there is a need for more experimental studies on cAMP and Adcyl in the regulation of glomerular filtration.

\section{Acknowledgments}

The authors are grateful to Maya H. Nisancioglu for critical reading of the paper. This research was supported by the Sigrid Juselius Foundation (J.P.), Finnish Cultural Foundation (J.P.), the Swedish Society of Medicine (J.P.), the Alice and Knut Wallenberg Foundation (K.T.) and NIH grant NS020498 (D.R.S.).

References

- 1 Tryggvason K, Patrakka J, Wartiovaara J: Hereditary proteinuria syndromes and mechanisms of proteinuria. N Engl J Med 2006;354:1387-1401.

2 Kestila M, Lenkkeri U, Mannikko M, et al: Positionally cloned gene for a novel glomerular protein - nephrin - is mutated in congenital nephrotic syndrome. Mol Cell 1998; 1:575-582.

- 3 Boute N, Gribouval O, Roselli S, et al: NPHS2, encoding the glomerular protein podocin, is mutated in autosomal recessive steroid-resistant nephrotic syndrome. Nat Genet 2000;24:349-354.

-4 Kaplan JM, Kim SH, North KN, et al: Mutations in ACTN4, encoding alpha-actinin-4, cause familial focal segmental glomerulosclerosis. Nat Genet 2000;24:251-256.

5 Kim JM, Wu H, Green G, et al: CD2-associated protein haploinsufficiency is linked to glomerular disease susceptibility. Science 2003;300:1298-1300 
6 Reiser J, Polu KR, Moller CC, et al: TRPC6 is a glomerular slit diaphragm-associated channel required for normal renal function. Nat Genet 2005;37:739-744.

7 Hinkes B, Wiggins RC, Gbadegesin R, et al: Positional cloning uncovers mutations in PLCE1 responsible for a nephrotic syndrome variant that may be reversible. Nat Genet 2006;38:1397-1405.

$\checkmark 8$ Winn MP, Conlon PJ, Lynn KL, et al: A mutation in the TRPC6 cation channel causes familial focal segmental glomerulosclerosis. Science 2005;308:1801-1804.

$\checkmark 9$ Pavenstadt H, Kriz W, Kretzler M: Cell biology of the glomerular podocyte. Physiol Rev 2003;83:253-307.

10 Faul C, Asanuma K, Yanagida-Asanuma E, et al: Actin up: regulation of podocyte structure and function by components of the actin cytoskeleton. Trends Cell Biol 2007;17:428437.

11 Wang H, Storm DR: Calmodulin-regulated adenylyl cyclases: cross-talk and plasticity in the central nervous system. Mol Pharmacol 2003;63:463-468.

-12 Endlich N, Endlich K: cAMP pathway in podocytes. Microsc Res Tech 2002;57:228231.

13 Lemieux LI, Rahal SS, Kennedy CR: PGE2 reduces arachidonic acid release in murine podocytes: evidence for an autocrine feedback loop. Am J Physiol Cell Physiol 2003; 284:C302-C309.
14 Martineau LC, McVeigh LI, Jasmin BJ, et al: p38 MAP kinase mediates mechanically induced COX-2 and PG EP4 receptor expression in podocytes: implications for the actin cytoskeleton. Am J Physiol Renal Physiol 2004;286:F693-F701.

15 Bek M, Nusing R, Kowark P, et al: Characterization of prostanoid receptors in podocytes. J Am Soc Nephrol 1999;10:2084-2093.

16 Sharma R, Lovell HB, Wiegmann TB, et al: Vasoactive substances induce cytoskeletal changes in cultured rat glomerular epithelial cells. J Am Soc Nephrol 1992;3:1131-1138.

17 Takemoto M, He L, Norlin J, et al: Largescale identification of genes implicated in kidney glomerulus development and function. EMBO J 2006;25:1160-1174.

18 Patrakka J, Ruotsalainen V, Ketola I, et al: Expression of nephrin in pediatric kidney diseases. J Am Soc Nephrol 2001;12:289296.

19 Wu ZL, Thomas SA, Villacres EC, et al: Altered behavior and long-term potentiation in type I adenylyl cyclase mutant mice. Proc Natl Acad Sci USA 1995;92:220-224.

20 Putaala H, Soininen R, Kilpelainen P, et al: The murine nephrin gene is specifically expressed in kidney, brain and pancreas: inactivation of the gene leads to massive proteinuria and neonatal death. Hum Mol Genet 2001;10:1-8.

21 Roselli S, Gribouval O, Boute N, et al: Podocin localizes in the kidney to the slit diaphragm area. Am J Pathol 2002;160:131-139.

-22 Patrakka J, Xiao Z, Nukui M, et al: Expression and subcellular distribution of novel glomerulus-associated proteins dendrin, Ehd3, Sh2d4a, Plekhh2, and 2310066E14Rik. J Am Soc Nephrol 2007;18:689-697.
23 Reiser J, von Gersdorff G, Loos M, et al: Induction of B7-1 in podocytes is associated with nephrotic syndrome. J Clin Invest 2004; 113:1390-1397.

24 Defer N, Best-Belpomme M, Hanoune J: Tissue specificity and physiological relevance of various isoforms of adenylyl cyclase. Am J Physiol Renal Physiol 2000;279:F400-F416.

$>25$ Bek MJ, Zheng S, Xu J, et al: Differential expression of adenylyl cyclases in the rat nephron. Kidney Int 2001;60:890-899.

-26 Mundel P, Heid HW, Mundel TM, et al: Synaptopodin: an actin-associated protein in telencephalic dendrites and renal podocytes. J Cell Biol 1997;139:193-204.

27 Rastaldi MP, Armelloni S, Berra S, et al: Glomerular podocytes contain neuron-like functional synaptic vesicles. FASEB J 2006: 20:976-978.

28 Bek MJ, Wahle S, Müller B, et al: Stra13, a prostaglandin E2-induced gene, regulates the cellular redox state of podocytes. FASEB J 2003;17:682-684.

29 Morita H, Yoshimura A, Inui K, et al: Heparan sulfate of perlecan is involved in glomerular filtration. J Am Soc Nephrol 2005;16: 1703-1710.

30 Ishola DA Jr, van der Giezen DM, Hahnel B, et al: In mice, proteinuria and renal inflammatory responses to albumin overload are strain-dependent. Nephrol Dial Transplant 2006;21:591-597.

31 Tam FW, Smith J, Agarwal S, et al: Type IV phosphodiesterase inhibitor is effective in prevention and treatment of experimental crescentic glomerulonephritis. Nephron 2000;84:58-66. 